\title{
¿Bandas elásticas o equipos de gimnasio para el entrenamiento de adultos mayores? Elastic bands or gym equipment for the training of older adults?
}

*Daniel Miranda-Aguilar, **Pablo Valdés-Badilla, ***Tomás Herrera-Valenzuela, ****Eduardo Guzmán-Muñoz, *****Braulio Henrique Magnani Branco, ${ }^{* * * *}$ Guillermo Méndez-Rebolledo, ******Antonio López-Fuenzalida

*Universidad Bernardo O’Higgins (Chile), **Universidad Autónoma de Chile (Chile), ***Universidad de Santiago de Chile (Chile), ****Universidad Santo Tomás (Chile), *****Centro Universitário de Maringá (Brasil), ******Pontificia Universidad Católica de Chile (Chile)

\begin{abstract}
Resumen. El objetivo del presente estudio fue comparar los efectos de seis semanas de entrenamiento de resistencia muscular con el uso de bandas elásticas (BE) respecto al uso de equipos tradicionales de gimnasio (ETG) sobre la fuerza máxima, composición corporal, prensión manual, presión arterial, glicemia sanguínea, agilidad, equilibrio dinámico, flexibilidad y potencia aeróbica máxima $\left(\mathrm{VO}_{2 \text { ico }}\right)$ en adultos mayores. Estudio experimental que evaluó dos grupos de adultos mayores, uno con BE ( $\mathrm{n}=7$ ) y otro con ETG ( $\mathrm{n}=5$ ). Se reportó en ambos grupos un aumento significativo de la fuerza máxima (BE: $p=0,043$ y ETG: $p=0,018), \mathrm{VO}_{2 \text { pico }}$ (BE: $p=0,008$ y ETG: $p=0,038$ ) y flexibilidad del tren inferior (BE: $p=0,021$ y ETG: $p=0,027$ ), y una reducción de la masa adiposa (BE: $p=0,028$ y ETG: $p=0,021$ ). En conclusión, ambos entrenamientos producen efectos similares sobre variables neuromusculares, antropométricas y metabólicas en adultos mayores. Por lo tanto, usar BE fue tan efectivo como los ETG para entrenar adultos mayores.
\end{abstract}

Palabras clave

Antropometría, fuerza muscular, condición física, ejercicio, adultos mayores, envejecimiento activo.

\begin{abstract}
The aim of the present study was to compare the effects of six weeks of resistance training with the use of elastic bands (EB) to the use of traditional gym equipment (TGE) on maximum strength, body composition, manual grip, blood pressure, glycemia blood, agility, dynamic balance, flexibility, and peak aerobic consumption in older adults. This is an experimental study that evaluated two groups of older adults, one with EB $(n=7)$ and another with TGE $(n=5)$. A significant increase in maximal strength (EB: $p=0.043$ and TGE: $p=0.018$ ), peak aerobic consumption (EB: $p=0.008$ and TGE: $p=0.038$ ) and lower train flexibility (EB: $p=0.021$ and TGE: $p=0.027$ ), and a decrease of fat mass (EB: $p=0.028$ and TGE: $p=0.021$ ) were found. In conclusion, both training produce similar effects on neuromuscular, anthropometric, and metabolic variables in older adults. Therefore, using EB showed as effective as ETG in the training of older adults.
\end{abstract}

Keywords. Anthropometry, muscle strength, physical fitness, exercise, elderly, active aging.

\section{Introducción}

La cantidad de adultos mayores (AM) va en aumento en todo el mundo (World Health Organization, 2012) y se espera que la región Sudamericana sea una de las de mayor proporción para el año 2050 (He, Goodkind, \& Kowal, 2016). En Chile, la situación es preocupante debido al incremento sostenido de AM el cual alcanza el 19,4\% de la población (Instituto Nacional de Estadisticas Chile, 2017), posicionando al país como uno de los de mayor envejecimiento a nivel global (He et al., 2016; World Health Organization, 2012).

El envejecimiento es el producto de diferentes cambios físicos, fisiológicos y funcionales (Valdés-Badilla, GutiérrezGarcía, Pérez-Gutiérrez, Vargas-Vitoria, \& López-Fuenzalida, 2019) que se asocian con mayor prevalencia de enfermedades crónicas (Ogawa, You, \& Leveille, 2016). Dicho proceso se caracteriza, entre otras cosas, por la pérdida de masa muscular y fuerza muscular (Enríquez-Reyna, Carranza Bautista, \& Navarro Orocio, 2019; Padilla Colón, Sánchez Collado, \& Cuevas, 2014). La pérdida de fuerza muscular aumenta el riesgo de caídas y la incidencia de fracturas, provocando una disminución de la independencia funcional, y al mismo tiempo, aumento de los gastos médicos y la mortalidad en la vejez (Colado et al., 2018; Colado \& Triplett, 2008; EnríquezReyna et al., 2019; Ogawa et al., 2016; Valdés-Badilla et al., 2019).

Diversas investigaciones demuestran que intervencio-

Fecha recepción: 14-06-19. Fecha de aceptación: 15-10-19

Pablo Valdés-Badilla

pablo.valdes@uautonoma.cl nes con actividad física que contemplan entrenamiento de resistencia muscular impactan positivamente sobre la fuerza, potencia muscular, y el control metabólico en los AM (Agner et al., 2018; Lima et al., 2018; Padilla Colón et al., 2014; Castro et al., 2019; Mosteiro-Muñoz \& Dominguez, 2017). Sin embargo, los programas de entrenamiento enfocados en la mejora de la resistencia muscular cuentan con limitaciones por el uso de sofisticados equipos de ejercicio que impiden el acceso a los AM, además de requerir capacitación para su uso (Bergquist, Iversen, Mork, \& Fimland, 2018). Por el contrario, se ha propuesto como una alternativa asequible el uso de bandas elásticas (BE) por su simplicidad, portabilidad, ligereza (Motalebi, Amirzadeh Iranagh, Mohammadi, \& Cheong, 2018; Soria-Gila, Chirosa, Bautista, Baena, \& Chirosa, 2015) y mayor nivel de activación muscular gracias a su modalidad inestable (Bergquist et al., 2018; Soria-Gila et al., 2015). A pesar de que las BE comparadas con los equipos tradicionales de gimnasio (ETG) demuestran una pequeña desventaja en la ganancia de fuerza (Bergquist et al., 2018; Colado \& Triplett, 2008; Lima et al., 2018), es probable que la selección de ejercicios con resistencia variable (p.e. BE) pueda ser mejor o tan efectiva como los realizados con ETG (Colado \& Triplett, 2008; Soria-Gila et al., 2015), debido a los beneficios otorgados por la resistencia variable a nivel del reclutamiento de unidades motoras (Melchiorri \& Rainoldi, 2011).

En este contexto, comparar el mismo programa de entrenamiento muscular utilizando BE respecto al uso de ETG y medir sus efectos sobre variables neuromusculares, antropométricas y metabólicas, podría servir de fundamento para implementar actividades más seguras y apropiadas a las características de los AM, con la intención de mejorar su 
capacidad física funcional (Lima et al., 2018). En este sentido, la presente investigación tiene por objetivo principal comparar los efectos de seis semanas de entrenamiento de resistencia muscular con el uso de BE respecto al uso de ETG sobre la fuerza máxima, composición corporal, prensión manual, presión arterial, glicemia sanguínea, agilidad, equilibro dinámico, flexibilidad y potencia aeróbica máxima $\left(\mathrm{VO}_{\text {2pico }}\right)$ en AM.

\section{Material y método}

Se realizó un estudio experimental con enfoque cuantitativo que consideró la aleatorización electrónica (https:// www.randomizer.org) de los participantes, distribuyéndolos en un grupo con BE (grupo experimental) y un grupo con ETG (grupo control).

\section{Participantes}

Veintiún AM de ambos sexos (4 hombres y 17 mujeres), pertenecientes al Centro Cultural de Santiago de Chile participaron inicialmente de la investigación, realizada entre los meses de octubre y noviembre del 2018. Los criterios de inclusión fueron: a) poseer 60 años o más; b) ser físicamente independiente. Se consideró independiente a la persona que contaba con un puntaje e» 43 puntos en el Examen de Medicina Preventiva del Adulto Mayor (Ministerio de Salud, 2013); c) contar con capacidad para comprender y seguir instrucciones de forma contextualizada mediante comandos simples; d) asistir al menos al 85\% de las sesiones de la intervención. Los criterios de exclusión fueron: a) presentar afecta- ciones musculoesqueléticas como lesiones agudas o crónicas; b) poseer reemplazo de miembros como uso de prótesis u órtesis; c) presentar una patología metabólica o pulmonar no controlada; $y$ d) presentar problemas de coagulación diagnosticado. Finalmente, quedaron 7 AM (1 hombre y 6 mujeres) para el grupo con BE (experimental) y $5 \mathrm{AM}$ (2 hombres y 3 mujeres) para el grupo con ETG (control). En la figura 1 se presenta la descripción gráfica del proceso de aleatorización de los participantes.

Todos los participantes fueron informados del propósito del estudio y firmaron un consentimiento informado que autoriza el uso de la información con fines científicos. El protocolo de investigación fue revisado y aprobado por el Comité Ético Científico de la Universidad Santo Tomás (N86/ 2018) y fue desarrollado siguiendo lo expuesto en la Declaración de Helsinki.

\section{Evaluaciones}

Las evaluaciones pre y post intervención se obtuvieron durante una semana y fueron recogidas en el siguiente orden: el día uno se ejecutaron las pruebas para valorar la composición corporal, glicemia sanguínea, presión arterial, agilidad, equilibro dinámico y flexibilidad. El día dos se evaluó la fuerza máxima. El día tres se determinó la fuerza de prensión manual y el día cuatro se realizó la medición de $\mathrm{VO}_{2 \text { pico }}$. Todas las evaluaciones fueron realizadas en un recinto acondicionado en temperatura $\left(18^{\circ}\right.$ a $\left.20^{\circ} \mathrm{C}\right)$ y espacio para la comodidad de los AM, además se les pidió a los dos grupos no modificar sus hábitos de alimentación, actividad física y sueño durante el periodo que duraba la intervención.

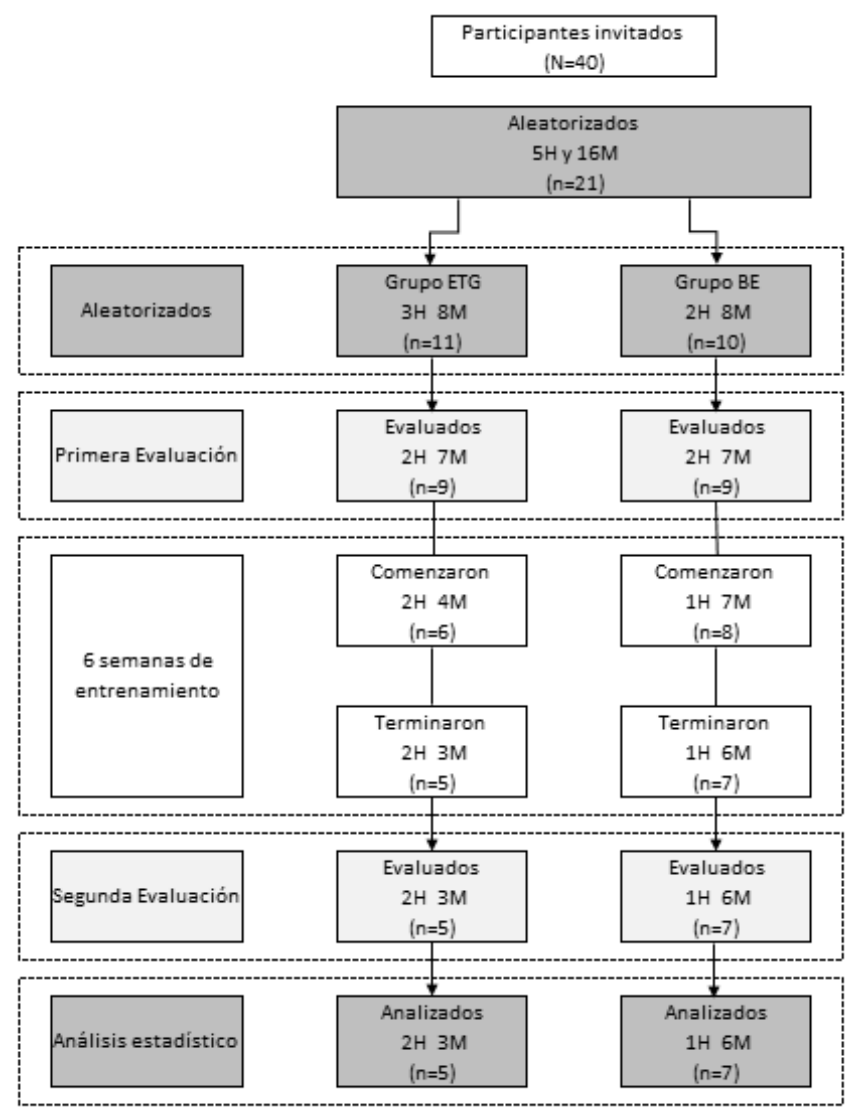

Figura 1.

Flujograma del proceso de reclutamiento y aleatorización de los participantes Abreviaturas: ETG: equipos tradicionales de gimnasio; BE: bandas elásticas; $\mathrm{H}$ : hombres; M: mujeres.

\section{Fuerza máxima}

Se obtuvo a través de la estimación de la repetición máxima (RM), siguiendo las recomendaciones para evaluar AM (Heyward, 2008). Para este estudio, se determinó la RM de forma indirecta utilizando pesos submáximos hasta el fallo muscular del participante, buscando menos de 10 repeticiones. La RM se calculó según la fórmula de Brzycki (1993):

$1 R M=P L /(1.0278-$ [RHF X 0.0278])

Dónde PL= Peso levantado y RHF= Repeticiones hasta el fallo.

Cada participante fue familiarizado con la evaluación y se les solicitó que en la ejecución dieran su máximo esfuerzo, el equipo utilizado fue de resistencia constante y supervisado por tres evaluadores entrenados para 
resguardar la seguridad de los AM y la correcta ejecución de los ejercicios (Heyward, 2008). Se estimó la RM con el ejercicio press de banco para el tren superior y con sentadilla paralela (rodillas en ángulo de $90^{\circ}$ ) para el tren inferior considerando las recomendaciones del Colegio Americano de Medicina del Deporte (Garber et al., 2011). Ambos ejercicios fueron obtenidos pre y post intervención con una máquina Smith multifunción (Tuffstuff, USA).

\section{Fuerza de prensión manual}

Para la medición de la fuerza de prensión manual se utilizó un dinamómetro hidráulico (Camry, modelo EH101, China) con la mano derecha e izquierda. La evaluación se realizó con los AM sentados, la columna alineada, hombros en aducción y sin rotación, codo en flexión de $90^{\circ}$ a un costado del cuerpo, antebrazo y muñeca en posición neutra. El participante realizó una fuerza de prensión manual máxima durante tres segundos, con reposo de un minuto entre cada repetición realizando dos intentos, donde el mejor de ambos intentos fue utilizado para los análisis posteriores, siguiendo recomendaciones de estudios previos (Mancilla, Ramos, \& Morales, 2016).

\section{Composición corporal}

Para identificar los cambios en masa muscular y masa adiposa, se registraron las siguientes dimensiones antropométricas: a) peso corporal en kg con báscula electrónica (Seca 769, Alemania; Precisión de 0,1 kg); b) estatura bípeda en cm con estadiómetro (Seca 220, Alemania; Precisión 0,1 cm); c) pliegues cutáneos en mm con cáliper (Slim Guide, China; precisión: 0,5 mm), a través de los pliegues tricipital, subescapular, supraespinal, abdominal, muslo frontal y pantorrilla medial; d) perímetros en cm con cinta métrica (Seca 201, Alemania; precisión 0,1cm) registrando el valor del brazo relajado, antebrazo máximo, tórax transverso, muslo máximo, muslo medial y pantorrilla máxima. Todas las medidas se realizaron de acuerdo a las recomendaciones de la Sociedad Internacional para Avances de la Cineantropometría (ISAK) (Marfell-Jones, Stewart, \& de Ridder, 2012) a través de un antropometrista nivel II (error técnico de medición: $0,8 \%$ para todas las variables evaluadas en los AM) de la ISAK. Mediante la técnica indirecta de fraccionamiento pentacompartimental propuesta por Ross and Kerr (1993) se determinó la masa muscular y la masa adiposa de todos los AM, utilizando el mismo modelo antropométrico pre y post intervención. Además, se calculó el índice de masa corporal (IMC) de cada AM dividiendo el peso corporal en kg por la estatura bípeda en $\mathrm{m}^{2}$.

\section{Presión arterial sistólica y diastólica}

Fue medida con un monitor de presión automático (08A, CONTEC, Alemania). La medición fue realizada por un técnico del área de la salud certificado, quien evaluó a los participantes contabilizando al menos 10 min de reposo previo en una posición sentada, con la espalda y brazos apoyados y las piernas sin cruzar, vaciando la vejiga urinaria en caso de necesitarlo. Además, se les solicitó a los participantes no realizar ejercicio físico, fumar e ingerir alcohol al menos 30 min antes de la medición y café o té $4 \mathrm{~h}$ antes (Ministerio de Salud, 2010). La primera medición se tomó en ambos brazos para identificar el brazo con mayor presión arterial, luego se tomaron dos mediciones en el brazo de mayor presión (habitualmente el dominante) agregando una tercera medición si la diferencia entre una medida y otra era mayor a $5 \mathrm{mmHg}$. Finalmente se registró el promedio de las medidas (Ministerio de Salud, 2010).

\section{Glicemia sanguínea}

A cada participante se le tomó pre y post intervención una muestra de sangre mediante un test rápido de punción capilar (Agner et al., 2018) para esto, se utilizó un Hemoglucotest (Accu-Chek ${ }^{\circledR}$ Performa, ROCHE, Alemania) en conjunto con las cintas reactivas del fabricante. Este procedimiento se realizó entre las 8:00 y 9:00 h, instruyendo a los participantes que asistieran con 8 a 12 h de ayuno. Dicha evaluación fue ejecutada por un profesional del área de la salud calificado para recolectar la muestra con los resguardos correspondientes.

\section{Potencia aeróbica máxima $\left(\mathrm{VO}_{2 \text { pica }}\right)$}

El $\mathrm{VO}_{2 \text { pico }}$ se estimó mediante la prueba indirecta de Rockport (George, Fisher, \& Verhs, 2007), el evaluado recorrió la distancia de 1609,3 m caminando lo más rápido posible sobre una cinta rodante (SportsArts, T652M, USA) con medidor de frecuencia cardíaca (H10, Polar, Finlandia). Los cálculos del $\mathrm{VO}_{2 \text { pico }}$ se realizaron en base a la edad, sexo, tiempo utilizado en recorrer la distancia y la frecuencia cardíaca al finalizar la prueba, utilizando la siguiente formula (Martínez López, 2017):

$\mathrm{VO}_{2 \text { pico }}(\mathrm{ml} / \mathrm{kg} / \mathrm{min})=132,6-(0,17 \mathrm{xPC})-(0,39 \mathrm{xED})+(6,31 \mathrm{x}$ S) $-(3,27 x \mathrm{~T})-(0.156 \mathrm{xFC})$

Dónde: $\mathrm{PC}=$ peso corporal, $\mathrm{ED}=\mathrm{edad}, \mathrm{S}=\operatorname{sexo}(0$ : mujeres; 1 : hombres), $\mathrm{T}=$ tiempo en min y valor decimal (00:00) y $\mathrm{FC}=$ frecuencia cardíaca en latidos/min.

\section{Agilidad y equilibrio dinámico}

Se utilizó el Timed Up and Go (TUG), prueba que es parte de la batería Senior Fitness Test (Rikli \& Jones, 2013). El TUG original utiliza un circuito de 2,44 m (8 pies) (Rikli \& Jones, 2013), sin embargo, se siguieron las instrucciones de los manuales propuestos por el Ministerio de Salud (2013) de Chile. En dicha versión el participante se desplaza por una pista previamente trazada a tres $\mathrm{m}$ de distancia. Se midió el tiempo que demoró el AM desde que despega la espalda de la silla, gira por detrás del cono y regresa hasta retomar la posición inicial (sentado en la silla). La prueba fue supervisada por un profesional del área de las ciencias de la actividad física familiarizado con el protocolo de la prueba y se consideró el menor tiempo, después de dos intentos, con un descanso de 10 min entre intentos.

\section{Flexibilidad}

Para evaluar la flexibilidad de los AM se utilizaron dos pruebas que forman parte del Senior Fitness Test: Chair-Sit and Reach-Test para medir la flexibilidad del tren inferior y Back Scratch Test para el tren superior (Rikli \& Jones, 2013). Ambas pruebas fueron evaluadas y dirigidas por un profesional del área de las ciencias de la actividad física familiarizado sobre los protocolos de las evaluaciones quien utilizó una cinta métrica (Seca 201, Alemania; precisión 0,1cm) para 
obtener el valor del mejor intento de cada prueba, después de dos repeticiones realizadas, con un descanso de 2 min entre intentos. Para el Chair-Sit and Reach-Test el participante se ubicó sentado en una silla, extendió la rodilla dominante apoyando el talón en el suelo y dejó la otra rodilla en $90^{\circ}$ con la planta apoyada en el suelo e intentó tocarse la punta del pie con ambas manos extendidas, manteniendo la posición. El evaluador midió la cantidad de cm (+ o -) entre los dedos extendidos y la punta de los pies. Para el Back Scratch Test el participante colocó una mano sobre el hombro y una hacia la mitad de la espalda manteniendo la posición. El evaluador midió la cantidad de cm (+ o -) entre los dedos anulares extendidos de ambas manos.

\section{Intervención}

El programa de entrenamiento de resistencia muscular estuvo en concordancia con las recomendaciones del Colegio Americano de Medicina del Deporte (Garber et al., 2011). Además, siguió las sugerencias y consideraciones de los modelos de intervención utilizados en estudios previos para la ganancia de fuerza muscular en AM (Bergquist et al., 2018; Colado et al., 2018; Colado \& Triplett, 2008; Lima et al., 2018). Las características iniciales de la muestra se aprecian en la tabla 1.

\begin{tabular}{|c|c|c|c|}
\hline \multicolumn{2}{|c|}{ FTC $(1-5)$} & ETG $(n=5)$ & \\
\hline & Media (DE) & Media (DE) & Valor $\mathrm{p}$ \\
\hline Edad & $67,285(4,208)$ & $67,200(2,167)$ & 0,970 \\
\hline Estatura bípeda & $155,728(6,049)$ & $159,940(9,611)$ & 0,371 \\
\hline Peso corporal & $72,928(11,293)$ & $75,200(14,896)$ & 0,769 \\
\hline IMC & $29,975(3,592)$ & $29,256(4,059)$ & 0,752 \\
\hline
\end{tabular}

\section{Sesiones de adaptación}

Previo a la intervención se realizaron dos sesiones de adaptación. La primera fue de familiarización y la segunda estimó el esfuerzo de cada AM según la escala de percepción del esfuerzo OMNI-RES (Resistance Exercise Scale). La distribución específica de estas sesiones fue:

Para la sesión de familiarización, los AM utilizaron cada uno de los implementos para conocer su resistencia y corregir la técnica respecto a los ejercicios seleccionados para la intervención (Motalebi et al., 2018). Para el trabajo con BE se utilizaron bandas (Thera-Band, USA) de $1,5 \mathrm{~m}$ por $12,5 \mathrm{~cm}$ de diferentes colores (amarillo, rojo, verde, azul y negro) según el nivel de resistencia, para aumentar o disminuir el ancho de agarre de las BE se utilizó como referencia el protocolo de Colado and Triplett (2008), adaptando el material de 1,5 m de la siguiente manera: se marcaron 25 puntos de referencia simétricos a 2,5 cm de distancia desde el punto central de la BE. Cada punto recibió un valor que disminuyó en un punto cada vez que se alejaba del centro de la BE, comenzando con 25; siendo el valor más alto un ancho de agarre más corto. Para el caso de los ETG, se utilizó una máquina Smith multifunción (TuffStuff, USA), una polea cruzada (TuffStuff, USA), una prensa inclinada en $45^{\circ}$ (TuffStuff, USA) y mancuernas de uno, 3,5, 4,5, 6,5, 9, 11, 13,5y $16 \mathrm{~kg}$ (TuffStuff, USA).

La segunda sesión estimó la percepción del esfuerzo de cada AM, debido a considerar que el valor de la RM que se obtuvo en la semana de evaluación no es aplicable directa- mente a las BE, por esta razón, se utilizaron las recomendaciones de Colado and Triplett (2008) igualando la intensidad de los ejercicios, ajustando el peso en los ETG y los diferentes agarres y colores en las BE para lograr un número igual de repeticiones. Por lo tanto, para las BE y ETG se determinaron 20 repeticiones máximas equivalentes a la clasificación de 10 puntos en la escala OMNI-RES, los participantes fueron animados a completar las repeticiones solicitadas hasta su máximo esfuerzo, para este proceso se estimaron tres a ocho intentos con descansos de dos min entre intento. Una vez obtenido el máximo esfuerzo se les otorgó nuevamente dos min de descanso y se les solicitó que repitieran el ejercicio con la carga obtenida para asociar el nivel de esfuerzo en los diferentes grupos musculares con un nivel de 10 puntos en la escala OMNI-RES y se registró el color y agarre para el grupo con BE o el peso en kg para el grupo con ETG como valores objetivos para la intervención. Luego mediante una carga exageradamente más liviana completaron nuevamente 20 repeticiones y se les pidió asociar este esfuerzo con la escala OMNI-RES.

\section{Sesiones de entrenamiento}

La intervención consistió en seis semanas (12 sesiones) de entrenamientos supervisados y distribuidos en dos sesiones semanales en días alternos. Cada sesión duró 60 min, comenzando con 10 min de calentamiento a través de ejercicios aeróbicos y de movilidad articular, seguido de 40 min de un programa de resistencia muscular que consideró ejercicios para el tren superior y tren inferior con descanso activo y finalizó con 10 min de vuelta a la calma por medio de ejercicios de flexibilidad estática y dinámica. Dicho entrenamiento se realizó con ambos grupos de estudio (experimental con BE y control con ETG), diferenciándose sólo por el tipo de implementación. Para garantizar que los programas de entrenamiento fuesen equivalentes se eligieron ejercicios dinámicos con características de estabilización similar, buscando abarcar grandes grupos musculares con movimientos lentos de dos segundos en contracción concéntrica y cuatro segundos en contracción excéntrica (Colado \& Triplett, 2008). Los ejercicios de tren superior y tren inferior se distribuyeron de la siguiente manera:

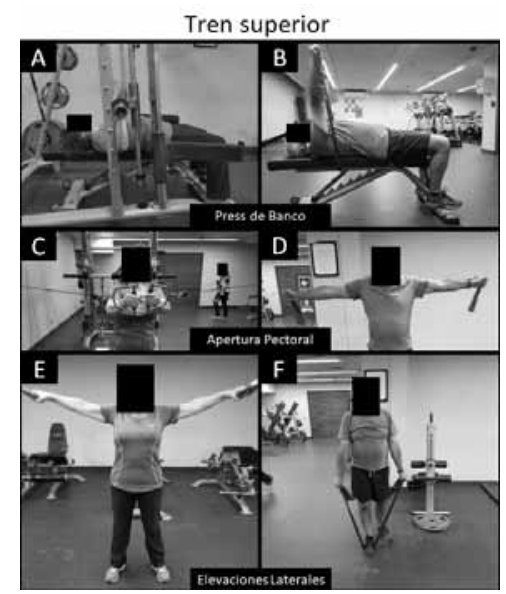

Figura 2.

Imágenes de los ejercicios realizados para el tren superior utilizando bandas elásticas y equipos tradicionales de gimnasio

Abreviaturas: A: press de banco con equipos tradicionales de gimnasio; B: press de banco con banda elástica; C: apertura lateral con equipos tradicionales de gimnasio; D: apertura lateral con banda elástica; E: elevaciones laterales con equipos tradicionales de gimnasio; F: elevaciones laterales con banda elástica. 


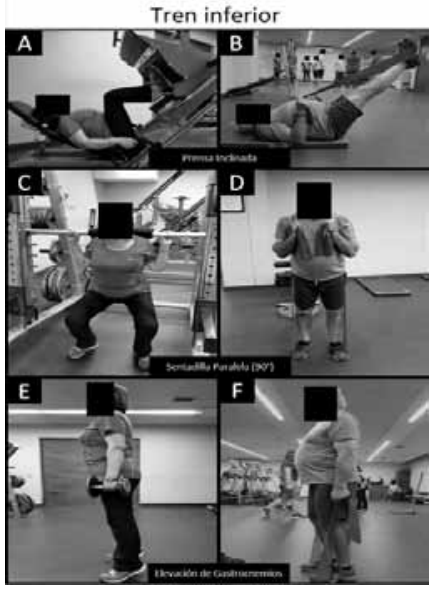

Figura 3.

Imágenes de los ejercicios realizados para el tren inferior utilizando bandas elásticas y equipos tradicionales de gimnasio

Abreviaturas: A: prensa inclinada con equipos tradicionales de gimnasio; B: prensa inclinada con banda elástica; C: sentadilla paralela $90^{\circ}$ con equipos tradicionales de gimnasio; D: sentadilla paralela $90^{\circ}$ con banda elástica; E: elevación de gastrocnemios con equipos tradicionales de gimnasio; F: elevación de gastrocnemios con banda elástica.

Los ejercicios para el tren superior fueron press de banco, apertura pectoral y elevaciones laterales. El press de banco se realizó en posición decúbito supino en un banco con la máquina Smith multifunción (TuffStuff, USA) para el grupo con ETG; el grupo con BE se ubicó decúbito supino en un banco fijando el implemento bajo el banco. La apertura pectoral se realizó de pie con una máquina de polea cruzada para el grupo con ETG; mientras que el grupo con BE apoyó el implemento en un soporte manteniendo la distancia de ejecución. Por último, las elevaciones laterales se desarrollaron de pie con mancuernas para el grupo con ETG; por su parte el grupo con BE, fijó la banda en un pie para ejecutar el movimiento. La figura 2 exhibe las imágenes de los ejercicios realizados por los AM para el tren superior.

Los ejercicios para el tren inferior fueron sentadilla paralela $\left(90^{\circ}\right)$, prensa de pierna inclinada y elevación de gastrocnemios. Las sentadillas paralelas $\left(90^{\circ}\right)$ se realizaron en posición de pie con la máquina Smith multifunción (TuffStuff, USA) para el grupo con ETG; mientras que el grupo con BE, utilizó de referencia la mitad de cada BE para pisarla con ambos pies y apoyaron los extremos del implemento en los hombros, realizando flexión de rodillas a $90^{\circ}$. La prensa de pierna inclinada se realizó con una prensa inclinada en $45^{\circ}$ para el grupo con ETG y el grupo con BE se colocó de cubito supino con una flexión de cadera de $45^{\circ}$ sobre una colchoneta, luego apoyaron la mitad de la $\mathrm{BE}$ entre ambos pies y tomaron ambos extremos del implemento con las manos y fijaron los brazos en el suelo, para realizar la extensión y flexión de rodilla. Por último, la elevación de gastrocnemios se realizó de pie con una mancuerna a cada lado del cuerpo para el grupo con ETG; y en la misma posición trabajó el grupo con BE, utilizando de referencia la mitad del implemento y tomando los extremos con las manos. La figura 3 exhibe las imágenes de los ejercicios realizados por los AM para el tren inferior.

La progresión de la carga se realizó utilizando como referencia la escala OMNI-RES (Colado et al., 2018), considerando los valores obtenidos por los AM en la evaluación inicial. Entre la primera y la tercera semana se mantuvo la intensidad al 50\% (5 puntos) de la escala OMNI-RES, mientras que entre la cuarta y la sexta semana se consideró una intensidad del 70\% (7 puntos). El volumen de trabajo se distribuyó de acuerdo al grupo de ejercicios, de esta manera para el tren superior se realizaron dos series entre la primera y cuarta semana, y tres series entre la quinta y sexta semana. Por su parte, para los ejercicios del tren inferior se realizaron dos

Tabla 2.

Efectos del entrenamiento de resistencia muscular con bandas elásticas y equipos tradicionales de gimnasio sobre la fuerza de los adultos mayores

\begin{tabular}{|c|c|c|c|c|c|c|c|}
\hline Variable & Segmento & Grupo & $\mathrm{n}$ & $\begin{array}{c}\text { Pre } \\
\text { Media (DE) }\end{array}$ & $\begin{array}{c}\text { Post } \\
\text { Media (DE) }\end{array}$ & $p$ & $T E$ \\
\hline \multirow{5}{*}{$\begin{array}{l}\text { Fuerza } \\
\text { máxima } \\
\text { (kg) }\end{array}$} & Tren & $\mathrm{BE}$ & 7 & $20,143(9,245)$ & $24,000(10,083)$ & 0,449 & 0,398 ף \\
\hline & superior & ETG & 5 & $29,400(14,791)$ & $28,400(12,895)$ & 0,394 & $0,072 \dagger$ \\
\hline & Tren & $\mathrm{BE}$ & 7 & $41,429(17,348)$ & $67,286(16,977)$ & $0,018^{*}$ & $1,506 ?$ \\
\hline & Inferior & ETG & 5 & $46,600(13,685)$ & $74,600(17,615)$ & $0,043^{*}$ & 1,775 ? \\
\hline & Mano & $\mathrm{BE}$ & 7 & $27,014(6,716)$ & $27,386(5,392)$ & 0,671 & $0,061 \dagger$ \\
\hline \multirow{3}{*}{$\begin{array}{c}\text { Fuerza } \\
\text { prensil (kg) }\end{array}$} & Derecha & ETG & 5 & $29,460(9,512)$ & $32,580(9,891)$ & $0,001^{*}$ & 0,321 ฯ \\
\hline & Mano & $\mathrm{BE}$ & 7 & $24,000(4,465)$ & $25,586(4,672)$ & 0,214 & 0,347 ศ \\
\hline & Izquierda & ETG & 5 & $28,300(7,866)$ & $30,360(9,894)$ & 0,156 & 0,23 \\
\hline
\end{tabular}
significativa $p<0,05$. ๆ: efecto pequeño $\dagger$ : efecto moderado; ? efecto fuerte.

Tabla 3.

Efectos del entrenamiento de resistencia muscular con bandas elásticas y equipos tradicionales de gimnasio sobre la composición corporal de los adultos mayores

\begin{tabular}{|c|c|c|c|c|c|c|}
\hline Variable & Grupo & $\mathrm{n}$ & $\begin{array}{c}\text { Pre } \\
\text { Media (DE) }\end{array}$ & $\begin{array}{c}\text { Post } \\
\text { Media (DE) } \\
\end{array}$ & $p$ & $T E$ \\
\hline eso & $\mathrm{BE}$ & 7 & $72,929(11,293)$ & $72,229(10,880)$ & 0,069 & 0,063 \# \\
\hline corporal (kg) & ETG & 5 & $75,200(1$ & $75,100(14$ & 0,756 & 0,006 ฯ \\
\hline \multirow{2}{*}{ IMC $\left(\mathrm{kg} / \mathrm{m}^{2}\right)$} & $\mathrm{BE}$ & 7 & $29,975(3$, & 29,821 & 0,230 & 0,043 ㄸ \\
\hline & ETG & 5 & $29,256(4,0$ & $29,272(3,896)$ & 0,904 & $0,004 \pi$ \\
\hline Masa & $\mathrm{BE}$ & 7 & $29,020(4,192)$ & $29,005(3,813)$ & 0,398 & 0,003 व \\
\hline muscular (kg) & ETG & 5 & $29,342(8,055)$ & $32,498(7,492)$ & 0,138 & 0,405 ศ \\
\hline Masa & $\mathrm{BE}$ & 7 & 20,615 & $18,640(3,859)$ & 028* & 43 \\
\hline
\end{tabular}

BE: bandas elásticas; ETG: equipos tradicionales de gimnasio; IMC: índice de masa corporal; n: número de casos; DE: desviación estándar; $p$ : significancia estadística; TE: tamaño del efecto. * La comparación es significativa $p<0,05$. ๆ: efecto pequeño $\dagger$ : efecto moderado; ? efecto fuerte.

Tabla 4.

Efectos del entrenamiento de resistencia muscular con bandas elásticas y equipos tradicionale de gimnasio sobre la condición física y variables metabólicas de los adultos mayores

\begin{tabular}{|c|c|c|c|c|c|c|c|}
\hline Variable & Segmento & Grupo & $n$ & Pre & Post & & $T E$ \\
\hline \multirow{2}{*}{$\begin{array}{c}\mathrm{VO}_{\text {2pico }} \\
(\mathrm{ml} / \mathrm{kg} / \mathrm{min})\end{array}$} & \multirow[b]{2}{*}{$\mathrm{n} / \mathrm{a}$} & $\mathrm{BE}$ & 7 & $\begin{array}{c}\text { Media (DE) } \\
-9,648(9,676)\end{array}$ & $\begin{array}{l}\text { Media (DE) } \\
2,890(6,109)\end{array}$ & $0,008^{*}$ & .549 \\
\hline & & ETG & 5 & $-0,756(25,673)$ & $16,702(15,795)$ & $0,038^{*}$ & 0,819 \\
\hline \multirow{3}{*}{ TUG (s) } & \multirow[b]{2}{*}{$\mathrm{n} / \mathrm{a}$} & $\mathrm{BE}$ & 7 & $6,652(0,381)$ & $6,102(0,758)$ & $0,043^{*}$ & \\
\hline & & ETG & 5 & $7,136(2,095)$ & $6,482(1,421)$ & 0,138 & 0,376 \\
\hline & 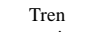 & $\mathrm{BE}$ & 7 & $-5,000(6,137)$ & $-4,857(6,414)$ & 0,895 & 0,022 \# \\
\hline \multirow{3}{*}{ Flexibilid } & superior & ETG & 5 & $-1,900(6,188)$ & $0,4(6,024)$ & 0,406 & 0,376 \\
\hline & Tren & $\mathrm{BE}$ & 7 & $4,785(7,392)$ & $10,142(7,641)$ & $0,021 *$ & 0,712 \\
\hline & inferior & ETG & 5 & $9,300(7,5$ & $11,500(6,837)$ & $0,027 *$ & 0,305 \\
\hline \multirow{3}{*}{$\begin{array}{c}\text { Glicemia } \\
\text { sanguínea (mg/dl) }\end{array}$} & \multirow[b]{2}{*}{$\mathrm{n} /$} & $\mathrm{BE}$ & 7 & $95,286(5,056)$ & $93,714(5,822)$ & 0,344 & 0,2 \\
\hline & & ETG & 5 & $99,800(7,049)$ & $98,200(2,280)$ & 0,686 & 0,3 \\
\hline & \multirow[b]{2}{*}{ tont } & $\mathrm{BE}$ & 7 & $130,191(11,205)$ & $123,380(12,034)$ & 0,124 & 0,58 \\
\hline \multirow{3}{*}{$\begin{array}{l}\text { Presión arterial } \\
\text { (mm Hg) }\end{array}$} & & ETG & 5 & $126,134(25,939)$ & $112,666(14,596)$ & 0,118 & 0,6 \\
\hline & \multirow{2}{*}{ Diastólica } & $\mathrm{BE}$ & 7 & $71,713(7,419)$ & $72,287(7,266)$ & 0,869 & 0,078 \\
\hline & & ETG & 5 & $65,932(10,963)$ & $63,132(9,729)$ & 0,211 & 0 , \\
\hline
\end{tabular}

BE: bandas elásticas; ETG: equipos tradicionales de gimnasio; n: número de casos; DE: desviación estándar; $p$ : significancia estadística; TE: tamaño del efecto. TUG: Timed Up and Go; n/a: no aplica * La comparación es significativa $p<0,05$. ף: efecto pequeño $\dagger$ : efecto moderado; ? efecto fuerte.

Tabla 5.

Diferencia entre el entrenamiento de resistencia muscular con bandas elásticas versus equipos tradicionales de gimnasio

\begin{tabular}{|c|c|c|c|c|c|}
\hline Variable & Segmento & Evaluación & $\begin{array}{l}\text { Porcentaje } \\
\text { de cambio }\end{array}$ & $T E$ & $\begin{array}{c}\text { BE vs ETG } \\
p\end{array}$ \\
\hline \multirow{5}{*}{ Fuerza máxima (kg) } & Tren & Pre & $-31,486$ & $0,75 \dagger$ & 0,209 \\
\hline & Superior & Post & $-15,492$ & $0,38 \pi$ & 0,521 \\
\hline & Tren & Pre & $-11,096$ & $0.33 \pi$ & 0,755 \\
\hline & Inferior & Post & $-9,804$ & $0,422 \dagger$ & 0,343 \\
\hline & Mano & Pre & $-8,302$ & 0,297 ศ & 0,611 \\
\hline \multirow{3}{*}{ Fuerza prensil (kg) } & Derecha & Post & $-15,942$ & $0,652 \dagger$ & 0,266 \\
\hline & Mano & Pre & $-15,194$ & $0,672 \dagger$ & 0,253 \\
\hline & Izquierda & Post & $-15,724$ & $0,617 \dagger$ & 0,286 \\
\hline \multirow{2}{*}{$\mathrm{VO}_{2 \text { pico }}(\mathrm{ml} / \mathrm{kg} / \mathrm{min})$} & & Pre & 1176,19 & $0,458 \dagger$ & 0,416 \\
\hline & n/a & Post & $-82,696$ & 1,153 ? & 0,059 \\
\hline \multirow{3}{*}{ TUG (s) } & & Pre & $-6,782$ & 0,321 ฯ & 0,268 \\
\hline & n/a & Post & $-5,862$ & $0,333 \pi$ & 0,876 \\
\hline & Tren & Pre & 163,157 & $0,503 \dagger$ & 0,410 \\
\hline \multirow{3}{*}{ Flexibilidad (cm) } & Superior & Post & $-1314,25$ & 0,844 ? & 0,182 \\
\hline & Tren & Pre & $-48,548$ & $0,603 \dagger$ & 0,326 \\
\hline & Inferior & Post & $-11,808$ & 0,187 ศ & 0,758 \\
\hline \multirow{3}{*}{$\begin{array}{l}\text { Glicemia sanguínea } \\
\text { (mg/dl) }\end{array}$} & & Pre & $-4,523$ & $0,735 \dagger$ & 0,149 \\
\hline & n/a & Post & $-4,568$ & 1,014 ? & 0,202 \\
\hline & & Pre & 3,216 & 0,203 ॠ & 0,717 \\
\hline \multirow{3}{*}{$\begin{array}{l}\text { Presión arterial } \\
\quad(\mathrm{mm} \mathrm{Hg})\end{array}$} & Sistolica & Post & 9,509 & 0,8 ? & 0,193 \\
\hline & & Pre & 8,768 & $0,617 \dagger$ & 0,299 \\
\hline & Diastonica & Post & 14,501 & 1,066 ? & 0,090 \\
\hline \multirow{2}{*}{ Peso corporal (kg) } & & Pre & $-3,019$ & 0,171 ฯ & 0,769 \\
\hline & II/d & Post & $-3,822$ & 0,219 q & 0,707 \\
\hline \multirow{2}{*}{$\mathrm{IMC}\left(\mathrm{kg} / \mathrm{m}^{2}\right)$} & & Pre & 2,457 & $0,187 \pi$ & 0,752 \\
\hline & n/a & Post & 1,875 & 0,149 ף & 0,801 \\
\hline \multirow{2}{*}{ Masa muscular (kg) } & & Pre & $-1,097$ & $0,05 \pi$ & 0,432 \\
\hline & $\mathrm{n} / \mathrm{a}$ & Post & $-10,748$ & $0,587 \dagger$ & 0,876 \\
\hline \multirow{2}{*}{ Masa adiposa (kg) } & & Pre & $-14,919$ & $0,741 \dagger$ & 0,239 \\
\hline & n/a & Post & $-1,187$ & $0,072 \pi$ & 0,909 \\
\hline
\end{tabular}

BE: bandas elásticas; ETG: equipos tradicionales de gimnasio; IMC: índice de masa corpora TE: tamaño del efecto; TUG: Timed Up and Go; vs: versus; p: significancia estadística. n/a: no
aplica. q: efecto pequeño t: efecto moderado; ? efecto fuerte. 
series entre la primera y segunda semana, y tres series entre la tercera y sexta semana. El número de repeticiones fue 20, las que se mantuvieron constantes para todos los ejercicios durante toda la intervención en ambos grupos.

\section{Análisis estadístico}

Se utilizó el programa SPSS 23.0 y la normalidad de las variables se verifico con el test Shapiro-Wilk. Para comparar las evaluaciones antes y después de la intervención, así como para la comparación entre los grupos, se aplicó la prueba $t$ de Student para los datos con distribución normal y la prueba de Wilcoxon para los datos con distribución no normal. El tamaño del efecto (TE) se calculó con la $d$ de Cohen (1992), considerando un efecto pequeño $(0,20-0,49)$, moderado $(0,50$ $0,79)$ o fuerte $(>0,80)$. En todos los casos, se estableció un valor de $p<0,05$.

\section{Resultados}

En la tabla 2 se exponen los resultados para las pruebas de fuerza. Se advierte que la fuerza máxima del tren inferior se incrementó significativamente en ambos grupos (BE: $p=0,043$; ETG: $p=0,018)$, mientras que el tren superior no reportó cambios significativos. Además, la fuerza prensil sólo mejoró significativamente en la mano derecha para el grupo con $\operatorname{ETG}(p=0,001)$.

Los cambios en la composición corporal de los AM se exponen en la tabla 3. Los únicos cambios significativos se aprecian en la reducción de la masa adiposa para ambos grupos (BE: $p=0,028$; ETG: $p=0,021$ ).

En la tabla 4 se exhiben los efectos de la intervención sobre la condición física y variables metabólicas. Se aprecia un aumento significativo en el $\mathrm{VO}_{2 \text { pico }}$ de ambos grupos (BE: $p=0,008$; ETG: $p=0,038$ ) y mejoras significativas en la flexibilidad del tren inferior (BE: $p=0,021$; ETG: $p=0,027$ ). Mientras que el tren superior, no reportó cambios significativos. La agilidad y equilibrio dinámico sólo mejoró significativamente en el grupo con $\mathrm{BE}(p=0,043)$.

La tabla 5 expone la diferencia entre el entrenamiento de resistencia muscular con el uso de BE respecto al uso de ETG en todas las variables analizadas, luego de seis semanas de intervención no se reportaron diferencias significativas entre los métodos.

\section{Discusión}

El objetivo principal del estudio fue comparar los efectos de seis semanas de entrenamiento de resistencia muscular con el uso de BE respecto al uso de ETG sobre la fuerza máxima, composición corporal, prensión manual, presión arterial, glicemia sanguínea, agilidad, equilibro dinámico, flexibilidad y $\mathrm{VO}_{2 \text { ico }}$ en $\mathrm{AM}$. El principal resultado señala que ambos sistemas de entrenamiento (BE y ETG) producen efectos similares sobre variables neuromusculares, antropométricas y metabólicas en los AM evaluados. Hecho coincidente con lo expuesto en estudios previos (Bergquist et al., 2018; Colado \& Triplett, 2008).

La fuerza máxima mejoró significativamente en el tren inferior de los AM, siendo más notorio en el grupo con BE. Situación que concuerda con el meta-análisis realizado por
Soria-Gila et al. (2015), donde se plantea que seis semanas de entrenamiento pueden mejorar hasta en un 35\% la fuerza máxima de los músculos extensores de rodilla. Por el contrario, el tren superior no reportó cambios significativos en los AM evaluados y sus resultados fueron contradictorios, por un lado el grupo con BE exhibió una tendencia de mejora, mientras que el grupo con ETG presentó una leve disminución de la fuerza. Estos resultados podrían relacionarse con los obtenidos en un estudio que utilizó electromiografía de superficie para evidenciar que los ejercicios con BE presentaron una mayor activación muscular de hombro (trapecio inferior, deltoides anterior y deltoides medio) en comparación con los ejercicios con mancuernas, lo cual, fue atribuido al estímulo neuromuscular que entrega la resistencia con carga variable (Bergquist et al., 2018). Además, se ha observado que el entrenamiento con dispositivos elásticos (bandas o tubos) puede mejorar la fuerza muscular (Aboodarda, Page, \& Behm, 2016), la velocidad de conducción de las unidades motoras (Melchiorri \& Rainoldi, 2011) y la amplitud electromiográfica de activación (Andersen et al., 2010). Estas mejoras están posiblemente asociadas a un mayor reclutamiento de unidades motoras rápidas en ejercicios con BE ejecutados hasta alcanzar la fatiga, lo que refleja una mejora en la activación neuromuscular en AM (Melchiorri \& Rainoldi, 2011).

Si bien la fuerza de prensión manual mejoró significativamente sólo en la mano derecha del grupo con ETG, el grupo con BE, mostró una tendencia a mejorar, situación considerada relevante debido que la fuerza de prensión manual es un marcador fiable para identificar fragilidad y mayor riesgo de mortalidad en los AM (García-Hermoso et al., 2018). Sin embargo, se esperarían resultados más satisfactorios con las $\mathrm{BE}$, ya que se requiere de un agarre en tensión constante (Bergquist et al., 2018). Es probable que el acotado tiempo de nuestra intervención explique los resultados conseguidos con las BE, por lo que futuras investigaciones podrían desarrollar protocolos de entrenamiento de resistencia muscular con mayor tiempo de aplicación.

En cuanto a la composición corporal, se advierte una reducción significativa en la masa adiposa de los dos grupos de intervención y, la masa muscular, sólo tiende a mejorar sin cambios significativos. Otras investigaciones han reportado una reducción significativa de la masa adiposa con BE, pero a la vez, buenos resultados sobre la hipertrofia muscular (Conlon et al., 2017; Fritz et al., 2018). Por otro lado, la masa muscular parece mejorar más con los ETG que con BE (Colado \& Triplett, 2008), sin embargo, no se encontraron diferencias significativas en este estudio para confirmarlo. Además, se ha planteado que mantener una alimentacion que cubra los requerimientos protéicos de los AM, ayudaría a mejorar la hipertrofia muscular (Bechshøft et al., 2017).

Pese a que la masa adiposa disminuye luego de la intervención con el uso de ambos implementos, el peso corporal y el IMC no presentaron ninguna variación importante. Al parecer la contribución del sistema nervioso fue más relevante que la hipertrofia muscular, esto se explicaría por la importancia que tienen los componentes neurales en la adaptación de la fuerza respecto al músculo esquelético en los AM durante las primeras semanas de entrenamiento (Bechshøft et al., 2017). Por lo tanto, seis semanas de entre- 
namiento muscular no serían suficientes para lograr cambios significativos a nivel de masa muscular, hecho que ya se ha planteado en investigaciones previas, incluso, con mayor tiempo de entrenamiento (Fritz et al., 2018). Por otra parte, es probable que considerar ejercicios que involucran grandes grupos musculares para el entrenamiento del tren inferior y superior, haya reducido los efectos de nuestra intervención, ya que otras investigaciones han conseguido mejoras significativas con ejercicios aislados para el pectoral mayor, deltoides y cuadriceps femoral (Bergquist et al., 2018; Pinho et al., 2018).

El $\mathrm{VO}_{\text {2pico }}$ aumentó significativamente en ambos grupos de intervención, hecho que coincide con investigaciones previas en AM que han incorporado ejercicios de fuerza muscular y resistencia aeróbica (Concha-Cisternas, GuzmanMuñoz, \& Marzuca-Nassr, 2017; Lima et al., 2018). En este contexto, se ha informado que el entrenamiento de resistencia produce mejoras sobre el flujo sanguíneo en hombres y mujeres de mediana edad, sin embargo, se desconoce si los efectos vasculares son similares en los AM (Egana, Reilly, \& Green, 2010). Por otra parte, se ha reportado que intervenciones con actividad física que incorporan entrenamiento de fuerza muscular y resistencia aeróbica son claves para el mantenimiento y mejora de la salud cardiovascular y metabólica en los AM (Valdés-Badilla et al., 2019), hecho que reforzaría los resultados encontrados en nuestra investigación que desarrollo un protocolo de entrenamiento basado en la resistencia muscular.

Respecto a la agilidad y equilibrio dinámico hubo una tendencia a disminuir el tiempo en el TUG en ambos grupos, pero sólo el grupo con BE reportó una disminución significativa. Esto podría ser atribuido a la modalidad inestable de las $\mathrm{BE}$, que producen mayor activación muscular respecto a los ETG (Bergquist et al., 2018), lo que permitiría un desempeño motor más eficiente durante la marcha. Esto coincide con la mejora de la funcionalidad motriz obtenida en otros estudios con AM (Concha-Cisternas et al., 2017; Motalebi et al., 2018). Tambien, el aumento de la fuerza muscular ha sido relacionado con la mejora del equilibrio (Pavol, Owings, Foley, \& Grabiner, 2002). Los cambios en la capacidad de equilibrio en respuesta a los aumentos de fuerza exhibidos por los AM, posiblemente se deban a un mayor desarrollo de las tasas de torque causadas por una mayor área de la seccioìn transversal de las fibras tipo II (Pavol et al., 2002). Hechos favorables, debido a que el equilibrio dinámico es una actividad compleja que involucra grandes grupos musculares, coordinación y función sensorial (Padilla Colón et al., 2014) y se relaciona con un menor riesgo de caídas en los AM (Valdés-Badilla et al., 2019).

Si bien existe una tendencia a mejorar la flexibilidad, sólo el tren inferior consigue cambios significativos en ambos grupos de intervención. Esto es coincidente con lo expuesto en otra investigación que reporta mejoras de la flexibilidad en AM (Concha-Cisternas et al., 2017), situación conveniente dado que la flexibilidad se asocia con mayor independencia para desarrollar las actividades de la vida diaria (ValdésBadilla et al., 2019).

En cuanto a la presión arterial y la glicemia sanguínea, estas tienden a disminuir en ambos grupos después de la intervención, sin presentar cambios significativos. Estudios previos han demostrado que la actividad física aumenta la captación de glucosa en AM a través de la estimulación de componentes musculares (Mazzeo et al., 1998) y posee efectos cardioprotectores bien determinados (Egana et al., 2010). Además, se indica que los ejercicios que involucran distintas capacidades físicas producen una reduccion significativa post ejercicio tanto en la presion diastólica como sistólica con una duración más prolongada que sólo ejercicio aeróbico (Ferrari et al., 2017). En ese contexto, es probable que el acotado tiempo de nuestra intervención sumado a los valores iniciales (normales) de glicemia sanguínea y presión arterial reportados por los participantes del estudio, dificultaran la mejora de dichas variables. No obstante, nuestra intervención ayudó a mantener los valores de glicemia sanguinea y presión arterial con tendencia a la baja.

Entre las posibles limitaciones del estudio se encuentran: a) no controlar la alimentación, lo que podría influir sobre la composición corporal, la presión arterial y glicemia sanguínea de los participantes; b) la amplitud de pesos (2,5 $\mathrm{kg}$ ) para programar los entrenamientos del grupo con ETG, lo que limitó la intensidad de los ejercicios; c) el pequeño número de participantes y la cantidad de semanas de intervención (seis) que podrían influir en los análisis estadísticos. Como principales fortalezas se encuentran: a) el diseño experimental del estudio que podría ser utilizado en investigaciones con muestras más amplias y más semanas de intervención; b) la aleatorización de los participantes, lo que aumenta la consistencia interna; c) el tipo de evaluaciones realizadas, que en su mayoría, cuentan con tablas normativas de respaldo; d) la planificación y desarrollo de la intervención, la cual contó con una progresión de la carga adecuada a las características y evaluaciones iniciales de los participantes, además de seguir las recomendaciones internacionales para el trabajo con AM (Bergquist et al., 2018; Colado et al., 2018; Colado \& Triplett, 2008; Conlon et al., 2017; Lima et al., 2018); hechos que en su conjunto, aumentan la validez externa del estudio.

\section{Conclusión}

No existen diferencias significativas entre el entrenamiento de resistencia muscular con BE y ETG sobre la fuerza máxima, composición corporal, prensión manual, presión arterial, glicemia sanguínea, agilidad, equilibro dinámico, flexibilidad y $\mathrm{VO}_{2 \text { pico }}$ en $\mathrm{AM}$. Además, tanto el entrenamiento con BE como con ETG producen mejoras significativas en $\mathrm{VO}_{\text {2pico }}$, flexibilidad del tren inferior y reducción de la masa adiposa, sin embargo, solamente el grupo con BE mejoró significativamente la agilidad y equilibrio dinámico. Por lo tanto, utilizar BE fue tan efectivo como los ETG para entrenar AM, pudiendo generar beneficios adicionales sobre la funcionalidad motriz.

Finalmente recomendamos el uso de BE para el diseño de programas gubernamentales de actividad física en AM como una estrategia más económica y accesible de promoción de la salud, las qué a su vez, podrían favorecer la capacidad física funcional, mejorar la calidad de vida, atenuar los impactos generados por las enfermedades crónicas y reducir los gastos en salud pública. En este sentido, la elección de los programas de entrenamiento podría estar en concor- 
dancia con la implementación del local, e incluso, considerar los intereses y experiencia de los usuarios, lo que favorecería la participación y adherencia de los AM con el ejercicio físico y consecuentemente, potenciar los resultados del entrenamiento.

\section{Agradecimientos}

A la Dirección de Vida Universitaria y la escuela de Pedagogía en Educación Física de la Universidad Bernardo O’Higgins, Chile.

\section{Referencias}

Aboodarda, S., Page, P., \& Behm, D. (2016). Muscle activation comparisons between elastic and isoinertial resistance: A meta-analysis. Clinical Biomechanics, 39, 52-61. https://doi.org/10.1016/j.clinbiomech.2016.09.008

Agner, V., Garcia, M., Taffarel, A., Mourão, C., da Silva, I., da Silva, S., . . . Lombardi J. (2018). Effects of concurrent training on muscle strength in older adults with metabolic syndrome: A randomized controlled clinical trial. Archives of gerontology and geriatrics, 75, 158-164. https:// doi.org/10.1016/j.archger.2017.12.011

Andersen, L., Andersen, C., Mortensen, O., Poulsen, O., Bjørnlund, I., \& Zebis, M. (2010). Muscle activation and perceived loading during rehabilitation exercises: comparison of dumbbells and elastic resistance. Physical therapy, 90(4), 538-549. https://doi.org/10.2522/ ptj.20090167

Bechshøft, R., Malmgaard-Clausen, N., Gliese, B., Beyer, N., Mackey, A., Andersen, J., . . . Holm, L. (2017). Improved skeletal muscle mass and strength after heavy strength training in very old individuals. Experimental gerontology, 92, 96-105. https://doi.org/10.1016/ j.exger.2017.03.014

Bergquist, R., Iversen, V., Mork, P., \& Fimland, M. (2018). Muscle activity in upper-body single-joint resistance exercises with elastic resistance bands vs. free weights. Journal of human kinetics, 61(1), 5-13. https://doi.org/ 10.1515/hukin-2017-0137

Brzycki, M. (1993). Strength testing-predicting a one-rep max from reps-to-fatigue. Journal of Physical Education, Recreation \& Dance, 64(1), 88-90. https://doi.org/ 10.1080/07303084.1993.10606684

Castro Jimenéz, L. E., Gálvez Pardo, A. Y., Guzmán Quintero, G. A., \& Garcia Muñoz, A. I. (2019). Fuerza exlosiva en adultas mayores, efectos del entrenamiento en fuerza máxima. Retos: nuevas tendencias en educación física, deporte y recreación, 36, 64-68. https://recyt.fecyt.es/ index.php/retos/article/view/66715/42192

Cohen, J. (1992). A power primer. Psychological bulletin, 112(1), 155-159.

Colado, J., Pedrosa, F., Juesas, A., Gargallo, P., Carrasco, J., Flandez, J., ... Naclerio, F. (2018). Concurrent validation of the OMNI-Resistance Exercise Scale of perceived exertion with elastic bands in the elderly. Experimental gerontology, 103, 11-16. https://doi.org/10.1016/ j.exger.2017.12.009

Colado, J., \& Triplett, N. (2008). Effects of a short-term resistance program using elastic bands versus weight machines for sedentary middle-aged women. The Journal of Strength \& Conditioning Research, 22(5), 1441-1448. 10.1519/JSC.0b013e31817ae67a

Concha-Cisternas, Y., Guzman-Muñoz, E., \& Marzuca-Nassr, G. (2017). Efectos de un programa de ejercicio físico combinado sobre la capacidad funcional de mujeres mayores sanas en Atención Primaria de Salud. Fisioterapia, 39(5), 195-201.10.1016/j.ft.2017.03.002

Conlon, J., Newton, R., Tufano, J., Peñailillo, L., Banyard, H., Hopper, A., . . . Haff, G. (2017). The efficacy of periodised resistance training on neuromuscular adaptation in older adults. European journal of applied physiology, 117(6), 1181-1194. https://doi.org/10.1007/s00421-017-3605-1

Egana, M., Reilly, H., \& Green, S. (2010). Effect of elasticband-based resistance training on leg blood flow in elderly women. Applied Physiology, Nutrition, and Metabolism, 35(6), 763-772. https://doi.org/10.1139/H10-071

Enríquez-Reyna, M. C., Carranza Bautista, D., \& Navarro Orocio, R. (2019). Nivel de actividad física, masa y fuerza muscular de mujeres mayores de la comunidad: Diferencias por grupo etario. Retos: nuevas tendencias en educación física, deporte y recreación, 35, 121-125. https:/ /recyt.fecyt.es/index.php/retos/article/view/59956/40927

Ferrari, R., Umpierre, D., Vogel, G., Vieira, P., Santos, L., de Mello, R., ... Fuchs, S. (2017). Effects of concurrent and aerobic exercises on postexercise hypotension in elderly hypertensive men. Experimental gerontology, 98, 1-7. https://doi.org/10.1016/j.exger.2017.08.012

Fritz, N., Juesas, Á., Gargallo, P., Calatayud, J., FernándezGarrido, J., Rogers, M., \& Colado, J. (2018). Positive effects of a short-term intense elastic resistance training program on body composition and physical functioning in overweight older women. Biological research for nursing, 20(3), 321-334. https://doi.org/10.1177/ 1099800418757676

Garber, C., Blissmer, B., Deschenes, M., Franklin, B., Lamonte, M., Lee, I., . . Swain, D. (2011). American College of Sports Medicine position stand. Quantity and quality of exercise for developing and maintaining cardiorespiratory, musculoskeletal, and neuromotor fitness in apparently healthy adults: guidance for prescribing exercise. Medicine and science in sports and exercise, 43(7), 13341359. 10.1249/MSS.0b013e318213fefb

García-Hermoso, A., Cavero-Redondo, I., Ramírez-Vélez, R., Ruiz, J., Ortega, F., Lee, D., \& Martínez-Vizcaíno, V.(2018). Muscular strength as a predictor of all-cause mortality in an apparently healthy population: a systematic review and meta-analysis of data from approximately 2 million men and women. Archives of physical medicine and rehabilitation, 99(10), 2100-2113.e5. https://doi.org/ 10.1016/j.apmr.2018.01.008

George, J., Fisher, A., \& Verhs, P. (2007). Tests y pruebas físicas. 4ta ed. Madrid: paidotribo.

He, W., Goodkind, D., \& Kowal, P. (2016). U.S. Census Bureau, International Population Reports, P95/16-1, An Aging World: 2015. Washington, DC. Recuperado de: https:// www.census.gov/content/dam/Census/library/ publications/2016/demo/p95-16-1.pdf.

Heyward, V. (2008). Evaluación de la aptitud física y pres- 
cripción del ejercicio: Madrid: Panamericana.

Instituto Nacional de Estadisticas Chile. (2017). Censo de Población y Vivienda 2017. Santiago: Instituto Nacional de Estadísticas de Chile. Recuperado de: https:// redatam-ine.ine.cl/redbin/RpWebEngine.exe/ Portal?BASE=CENSO_2017\&lang=esp.

Lima, F., Camillo, C., Gobbo, L., Trevisan, L., Nascimento, W., Silva, B., ... Ramos, E. (2018). Resistance training using low cost elastic tubing is equally effective to conventional weight machines in middle-aged to older healthy adults: a quasi-randomized controlled clinical trial. Journal of sports science \& medicine, 17(1), 153. https:// www.ncbi.nlm.nih.gov/pmc/articles/PMC5844202/

Mancilla, E., Ramos, S., \& Morales, P. (2016). Fuerza de prensión manual según edad, género y condición funcional en adultos mayores Chilenos entre 60 y 91 años. Revista médica de Chile, 144(5), 598-603. http:// dx.doi.org/10.4067/S0034-98872016000500007

Marfell-Jones, M., Stewart, A., \& de Ridder, J. (2012). International standards for anthropometric assessment. http://hdl.handle.net/11072/1510

Martínez López, E. (2017). Pruebas de Aptitud Física. 2da ed. Madrid: Paidotribo.

Mazzeo, R., Cavanagh, P., Evans, W., Fiatarone, M., Hagberg, J., McAuley, E.. \& Startzell, J. (1998). El ejercicio y la actividad física en los adultos mayores. Medicine \& Science in Sports \& Exercise, 30(6), 992-1008.

Melchiorri, G., \& Rainoldi, A. (2011). Muscle fatigue induced by two different resistances: Elastic tubing versus weight machines. Journal of Electromyography and Kinesiology, 21(6), 954-959. https://doi.org/10.1016/ j.jelekin.2011.07.015

Ministerio de Salud. (2010). Guía Clínica hipertensión arterial primaria o esencial en personas de 15 años y más. Santiago: Ministerio de Salud de Chile. Recuperado de: https://www.minsal.cl/portal/url/item/ 7220fdc4341c44a9e04001011f0113b9.pdf.

Ministerio de Salud. (2013). Manual de Aplicación del Examen de Medicina Preventiva del Adulto Mayor. Santiago: Ministerio de Salud de Chile. Recuperado de: http:// w e b. m i n s a l . cl/ p or t a l/ u r l/ i t e m/ ab1f81f43ef0c2a6e04001011e011907.pdf.

Mosteiro-Muñoz, F. y Domínguez, R. (2017) Efectos del entrenamiento con sobrecargas isoinerciales sobre la función muscular / Effects of Inertial Overload Resistance Training on Muscle Function. Revista Internacional de Medicina y Ciencias de la Actividad Física y el Deporte, 17 (68), 757-773. https://doi.org/10.15366/ rimcafd2017.68.011

Motalebi, S., Amirzadeh J. Mohammadi, F., \& Cheong, L. (2018). Efficacy of Elastic Resistance Training Program for the Institutionalized Elderly. Topics in Geriatric Rehabilitation, 34(2), 105-111. https://doi.org/10.1097/ TGR.0000000000000179

Ogawa, E., You, T., \& Leveille, S. (2016). Potential benefits of exergaming for cognition and dual-task function in older adults: a systematic review. Journal of aging and physical activity, 24(2), 332-336. https://doi.org/10.1123/ japa.2014-0267

Padilla Colón, C., Sánchez Collado, P., \& Cuevas, M. (2014).
Beneficios del entrenamiento de fuerza para la prevención y tratamiento de la sarcopenia. Nutrición Hospitalaria, 29(5), 979-988. http://dx.doi.org/10.3305/ nh.2014.29.5.7313

Pavol, M., Owings, T., Foley, K., \& Grabiner, M. (2002). Influence of lower extremity strength of healthy older adults on the outcome of an induced trip. Journal of the American Geriatrics Society, 50(2), 256-262. https:// doi.org/10.1046/j.1532-5415.2002.50056.x

Pinho, J., Mezêncio, B., Hernandez, A., Bonato, J., Masuko, W., Soncin, R., ... Amadio, A. (2018). Moderate intensity is a better stimulus to induce quadripecs femoris power in elderly adults. Revista Brasileira de Medicina do Esporte, 24(3), 230-233. http://dx.doi.org/10.1590/1517869220182403181345.

Rikli, R., \& Jones, J. (2013). Senior fitness test manual. 2da ed. Champaign: Human Kinetics.

Ross, W., \& Kerr, D. (1993). Fraccionamiento de la Masa Corporal: Un Nuevo Método para Utilizar en Nutrición, Clínica y Medicina Deportiva-G-SE/Editorial Board/Dpto. PubliCE. https://g-se.com/fraccionamiento-de-la-masacorporal-un-nuevo-metodo-para-utilizar-en-nutricionclinica-y-medicina-deportiva-261-sa-Q57cfb27120415

Soria-Gila, M., Chirosa, I., Bautista, I., Baena, S., \& Chirosa, L. (2015). Effects of variable resistance training on maximal strength: a meta-analysis. The Journal of Strength \& Conditioning Research, 29(11), 3260-3270. 10.1519/ JSC.0000000000000971

Valdés-Badilla, P., Gutiérrez-García, C., Pérez-Gutiérrez, M., Vargas-Vitoria, R., \& López-Fuenzalida, A. (2019). Effects of physical activity governmental programs on health status in independent older adults: a systematic review. Journal of aging and physical activity, 27(2), 265-275. https://doi.org/10.1123/japa.2017-0396

World Health Organization. (2012). Good health adds life to years: Global brieffor World Health Day 2012. Geneva: World Health Organization. Recuperado de: http:// w w w. who.int / a ge ing / p ub lic a tion s / whd2012_global_brief/en/.

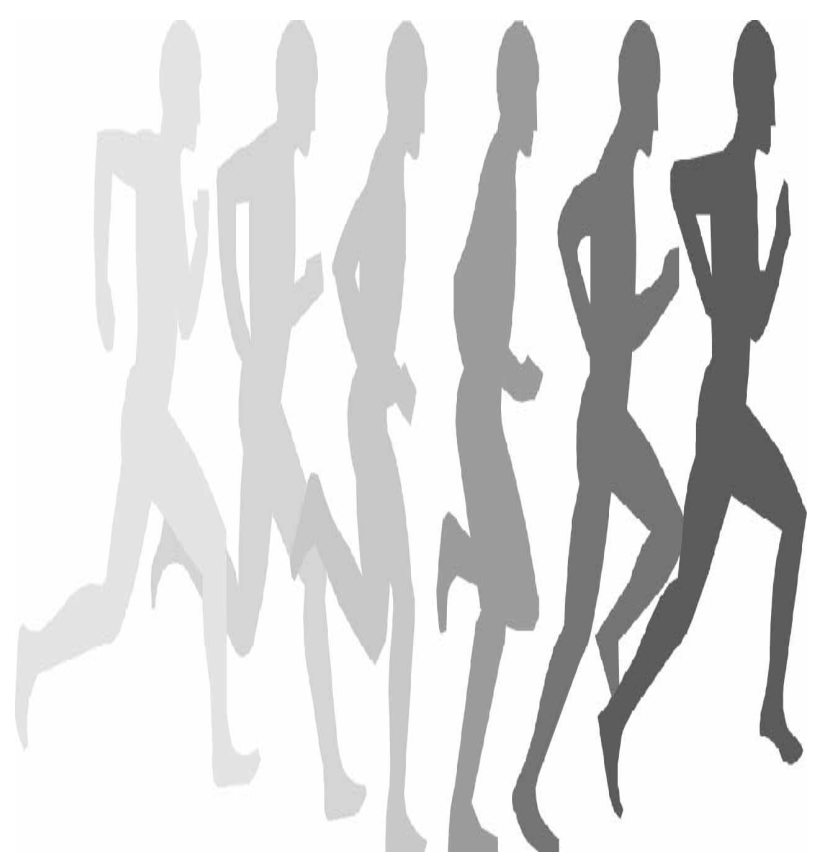

